\title{
Los estudios de caso: enseñanza de las matemáticas en una escuela de administración
}

\author{
Diego Alejandro Pérez ${ }^{1}{ }^{\circledR}$, Diana Victoria Jaramillo ${ }^{(0)}$, Flávia Asbahr ${ }^{\circledR}$ \\ Institución Universitaria Ceipa, Universidad de Antioquia - Colombia, Universidade Estadual Paulista-Júlio Mesquita Filho- Brasil
}

Autor de correspondencia:

11diego.perez@ceipa.edu.co

Recibido: 14 de octubre de 2019 Revisado: 30 de noviembre de 2019 Aprobado: 18 de febrero de 2020 Publicado: 24 de mayo de 2020

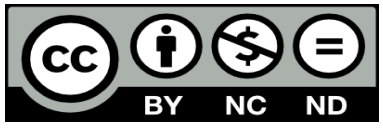

\section{Resumen}

Nuestro objetivo es mostrar el proceso de organización de enseñanza de tres profesores de matemáticas en una institución universitaria de administración, a partir de la creación colectiva de estudios de caso. El método dialéctico aportó elementos para comprender este proceso. A partir del trabajo colectivo, los profesores crearon un caso sobre oferta y demanda y expusieron un conocimiento matemático para aportar al análisis y a la solución de problemas empresariales. La fundamentación teórica de la investigación es la teoría histórico-cultural. En esta perspectiva, hablamos de organización de la enseñanza como objeto de la actividad del profesor; y de estudios de caso como metodología de enseñanza. Los instrumentos de producción de registros y datos utilizados fueron: mapas de caso, entrevistas semiestructuradas, audios, videos, ideogramas y mapas conceptuales. Analizamos que el proceso de escritura por parte de los profesores estuvo permeado por una intención de crear una forma propia de mostrar el conocimiento matemático, a partir de sus propias necesidades, experiencias y saberes.

Palabras clave: formación de docentes, enseñanza de las matemáticas, dialéctica, oferta y demanda, formación de administradores 


\title{
Case studies: teaching mathematics in a school of management
}

\begin{abstract}
Our aim is to show the organizational process of teaching of three math teachers in a management university institution, based on the collective creation of case studies. The dialectical method provided elements to understand this process. On the basis of collective work, the teachers made a case about supply and demand and presented a mathematical understanding to contribute to the analysis and solution of business problems. The theoretical foundation of the research is the historical-cultural theory. In this context, we understand the organization of teaching as the object of the teacher's activity, and case studies as teaching methodologies. Case maps, semi-structured interviews, audios, videos, ideograms, and concept maps were used as instruments of production of records and data. We analyzed that the writing process by the teachers was permeated by an intention of creating a particular way of showing mathematical knowledge, from their own needs, experiences and expertise.
\end{abstract}

Keywords: teacher training, mathematics teaching, dialectics, supply and demand, manager training

\section{Os estudos de caso: ensino das matemáticas numa escola de administração}

\section{Resumo}

Nosso objetivo é mostrar o processo de organização de ensino de três professores de matemática numa instituição universitária de administração, baseada na criação coletiva de estudos de caso. $\mathrm{O}$ método dialético contribuiu com elementos para compreender esse processo. A partir do trabalho coletivo, os professores criaram um caso de oferta e demanda e apresentaram um conhecimento matemático para contribuir com a análise e solução de problemas de negócios. O fundamento teórico da pesquisa é a teoria histórico-cultural. Nesta perspectiva falamos sobre a organização do ensino como objeto da atividade do professor, e de estudos de caso como metodologia de ensino. Os instrumentos de produção de registro e os dados utilizados foram: mapas de casos, entrevistas semiestruturadas, áudios, vídeos, ideogramas e mapas conceituais. Analisamos que o processo de escrita por parte dos professores esteve permeado pela intenção de criar sua própria maneira de mostrar o conhecimento matemático, a partir de suas próprias necessidades, experiências e saberes.

Palavras-chave: formação de professores, ensino das matemáticas, dialética, oferta e demanda, formação de administradores 


\section{Introducción ${ }^{1}$}

Las investigaciones centradas en procesos de reflexión e investigación sobre la actividad de enseñanza de profesores de matemáticas en programas universitarios se han desarrollado, como lo menciona Almeida (2012), en una menor proporción, comparadas con aquellas investigaciones sobre la formación inicial (Cedro, 2016; Jaramillo, 2003) y continuada de profesores que enseñan en contextos escolares (Araujo, 2003; Cadavid, 2017; Jiménez, 2005; Migueis, 2010; Moretti \& Moura, 2010; Polettini, 2000).

Nuestro objetivo es mostrar el proceso de organización de enseñanza de Andrés, Jacinto y José ${ }^{2}$, profesores del área de ciencias básicas en una institución universitaria de administración, a partir de la creación colectiva de estudios de caso. Dicho proceso se dio en el marco de una investigación doctoral, cuya pregunta orientadora fue: ¿cómo es el proceso de organización de la enseñanza de profesores que enseñan matemáticas en una escuela de administración, a partir de la creación colectiva de estudios de caso?

Para mostrar el camino recorrido por los profesores, comentaremos en primer lugar cuál fue la necesidad que fundamentó la investigación. En segundo lugar, mostraremos las principales características de los estudios de caso como una metodología de enseñanza que respondió a la necesidad expresada por los profesores. En tercer lugar, comentaremos lo que comprendimos sobre la formación de profesores que enseñan en contextos universitarios para, en cuarto lugar, mostrar la metodología de investigación utilizada y el análisis de una de las fases del trabajo de campo, la cual consistió, precisamente, en el proceso de creación del estudio de caso por parte de los profesores protagonistas.

\section{La organización de la enseñanza: objeto de la actividad del profesor}

La fundamentación teórica, epistemológica, gnoseológica y ontológica de la investigación doctoral es la teoría histórico-cultural. Esta perspectiva teórica tuvo su origen en los trabajos de Vygotski $(2001,2009)$, quien buscó en la filosofía marxista los fundamentos teóricos y metodológicos de una escuela psicológica que explicara la psiquis humana, centrándose en aspectos movilizadores de la conciencia.

En esta teoría, comprendemos la actividad como un proceso que se orienta hacia un objeto idealizado por el sujeto que, movido por un motivo, realiza acciones en el marco de condiciones contextuales que delimitan ciertas operaciones. En este sentido, comenta Leontiev (1988), las actividades son "procesos psicológicamente caracterizados por aquello a que el proceso, como un todo, se dirige (objeto), coincidiendo siempre con el objetivo que estimula al sujeto al ejecutar esa actividad, esto es, el motivo" (p. 68).

Así mismo, reconocemos el trabajo como una forma de actividad en la cual el hombre transforma la naturaleza y se transforma a sí mismo, mientras expresa su subjetividad en el objeto de dicha actividad. En otras palabras, el trabajo, como forma de actividad humana, es la posibilidad de que el hombre cree condiciones de subsistencia para sí mismo y, de manera colectiva, movilice el desarrollo de la sociedad. En este sentido, Leontiev (2004) explica que

\footnotetext{
1. Artículo derivado del proyecto de investigación doctoral titulado Organización de la enseñanza del profesor que enseña matemáticas en programas de administración: una posibilidad a partir de estudios de caso.

2. Nombres ficticios.
} 
el trabajo se efectúa en condiciones de actividad común colectiva, de modo que el hombre, en el seno de este proceso, no entra apenas en una relación determinada con la naturaleza, sino también con los otros hombres, miembros de una sociedad particular. Es solo por intermedio de esta relación con los otros hombres que el hombre se encuentra en relación con la naturaleza. (p. 80)

A propósito de estas ideas sobre actividad y trabajo, en la teoría histórico-cultural de la educación, comprendemos que el profesor, como trabajador que expresa su subjetividad en el objeto de su actividad de enseñanza, se encuentra ante una necesidad constante de apropiarse de metodologías que acerquen a sus estudiantes a un conocimiento - en este caso matemático-, el cual ha sido creado histórica y culturalmente. A propósito, Rigon, Asbahr y Moretti (2010) explican que

una de las responsabilidades del maestro es organizar situaciones didácticas que favorezcan el desarrollo, en el estudiante, de un querer aprender, una vez que este no es un valor natural, sino construido históricamente. Construir el motivo de aprender es fundamentalmente una función educativa que, dígase de paso, viene siendo menospreciada por gran parte de los educadores. (p. 31)

Así mismo, consideramos que esta actividad de organización de enseñanza de los profesores no solo debe responder a sus necesidades y expectativas, sino que, además, debe dar cuenta de los fundamentos pedagógicos, epistemológicos, gnoseológicos y ontológicos del proyecto educativo institucional de la universidad en la cual se da la actividad de enseñanza. A propósito, la institución universitaria de administración en la cual trabajan los protagonistas de la investigación se caracteriza por mostrar a través de núcleos problémicos (Mazo, 2011) el conocimiento gerencial para el desarrollo de competencias, como el análisis del entorno y el planteamiento y solución de problemas empresariales.

En el caso del área de ciencias básicas, cuyos cursos son matemáticas, estadística y métodos cuantitativos para los negocios, las metodologías de enseñanza usadas por los profesores se centran en tareas asociadas a la solución de problemas que, en la mayoría de los casos, responden a aspectos más próximos a la teoría que a la práctica. Como consecuencia, los profesores manifestaron una necesidad de crear una metodología que no solo diera cuenta de las expectativas formativas de la institución universitaria, sino que fuera coherente con aspectos de su subjetividad, tales como sus historias de vida, sus experiencias y su concepción sobre el conocimiento matemático.

Ante este panorama, formulamos una tesis doctoral cuyo objeto de estudio era el proceso de organización de enseñanza de los profesores del área de ciencias básicas de una institución universitaria. Dicha organización de enseñanza se centró en la creación colectiva de una metodología que, en los últimos años, ya se estaba utilizando en la institución, en las áreas específicas de la administración como el mercadeo, las finanzas, los negocios internacionales, la gestión humana y la contaduría. Esa metodología se conoce como estudios de caso o casos de enseñanza. Para efectos de la investigación, haremos uso del primer término.

\section{Los estudios de caso como metodología de enseñanza}

En las instituciones universitarias, los estudios de caso como metodología de enseñanza 
se han utilizado desde la segunda década del siglo XX como una posibilidad de dar soluciones a problemas reales a través de un análisis de las condiciones en las cuales las empresas se desenvuelven. Esta estrategia metodológica fue una respuesta a aquellos modelos pedagógicos centrados en la lectura pasiva de los estudiantes de problemas ajenos a ellos. De esta manera, la metodología de estudios de caso aportó a la construcción del conocimiento, no solo desde el análisis individual, sino también apoyado en la construcción colectiva de soluciones.

En esta investigación nos basamos en trabajos como los de Wassermann (1994), Ellet (2007; 2008), Stößlein y Kanet (2016), Arias, Betancourt, Garcés, Echeverri y Quirama (2017), entre otros, para definir al estudio de caso como narraciones - reales o ficticiasde situaciones empresariales en las cuales los estudiantes y los profesores analizan un problema, se cuestionan y debaten sobre sus posibles causas, proponen soluciones y elaboran conclusiones sobre la aplicación de estas soluciones a las problemáticas iniciales.

En esta definición resaltamos el carácter narrativo del estudio de caso, de modo que tanto estudiantes como profesores encuentren en el texto una historia, proveniente de situaciones reales o ficticias, y, a partir de sus propios saberes y experiencias, creen colectivamente condiciones de solución de estos problemas. De esta manera, como lo menciona Puchol (2005),

la metodología [de enseñanza] de estudios de caso no pretende que los formandos encuentren la solución ideal preestablecida, pues con frecuencia esa solución ideal no existe, o, alternativamente, se puede llegar a los mismos resultados por distintos caminos. La metodología [de enseñanza] estudios de caso huye así de las soluciones y opiniones dogmáticas, y pretende más bien el aprendizaje de los formandos en actitudes favorables a la resolución de problemas en equipo, favoreciendo la percepción de la realidad, la reflexión personal, la capacidad de análisis/síntesis y la asunción de riesgos. (p. 2)

Por otro lado, al caracterizar un estudio de caso, Wasserman (1994) explica que el texto creado debe contener: 1) una narración en la cual el estudiante y el profesor vean diálogos y expresiones que los motiven a continuar la lectura; 2) preguntas orientadoras, de modo que los estudiantes identifiquen cuál es el problema y, a través de un proceso de reflexión que se mueve entre lo individual y lo colectivo, se vislumbren las posibles soluciones; y 3) un espacio de discusión en la cual el profesor oriente el debate entre los estudiantes y proponga más preguntas dinamizadoras para, al final, recoger los aportes de los estudiantes, sintetizar las posibles soluciones y, si así lo considera el profesor, socializar la solución o soluciones más pertinentes.

Esta perspectiva de enseñanza que proponen los estudios de caso demanda que el profesor y los estudiantes asuman roles más activos, comparados con metodologías de enseñanza centradas únicamente en el profesor o en el estudiante. De esta manera, comprendemos que los estudios de caso, como metodología de enseñanza, ofrecen condiciones para que tanto estudiantes como profesores se vean reflejados en el objeto de su actividad de aprendizaje y su actividad de enseñanza, respectivamente; es decir, para que den cuenta de una actividad —en el sentido de la teoría histórico-cultural— al interior del aula de clase (Radford, 2016a; 2016b).

A propósito de este papel del profesor en el marco de la enseñanza con estudios de caso, 
en esta investigación consideramos que el profesor, como poseedor de un saber pedagógico y un saber específico, expresa a través de su actividad de organización de enseñanza su propia concepción sobre el conocimiento matemático y sobre la utilización de dicho conocimiento para no solo interpretar el mundo, sino también para transformarlo. En este sentido, la propuesta que les realizamos a los profesores Andrés, Jacinto y José consistió en apropiarse de una forma de creación de estudios de caso que concordara con el sentido personal que le atribuían a su propia actividad. En otras palabras, en esta investigación asumimos que el profesor era quien debía escribir los estudios de caso, ya que, como lo mencionan Fiorentini y Miorim (2001),

lo más importante y fundamental, en los procesos de innovación y producción de saberes, no es el modo en el que el profesor debe escribir, sino el hecho de que él necesita escribir sobre su trabajo. Necesita volver públicos sus saberes y experiencias. Haciendo esto, los profesores evitan que sus saberes se pierdan cuando dejen de enseñar. Además, es a través de la escritura que los profesores pueden influir en las políticas educativas y contribuir, a partir de la práctica, con los procesos de innovación curricular. (p. 44)

Así mismo, el profesor, para realizar un proceso de escritura que muestre su postura frente a su propia actividad, sobre los estudiantes, la institución donde trabaja y sobre el conocimiento matemático, está llamado a reflexionar sobre su propia actividad de enseñanza. Dicha reflexión, en un contexto universitario, es desde nuestro punto de vista uno de los fundamentos para la formación continuada de los profesores.

La formación de profesores que enseñan en la universidad y la reflexión sobre la propia actividad

En esta investigación comprendemos la importancia del entretejido de la trayectoria personal y profesional del profesor, lo cual lleva a sostener que él no es la suma de conocimientos sobre su saber específico, pedagógico y metodológico, sino que es un sujeto que se moviliza a partir de diversas experiencias de formación (Caballero \& Bolívar, 2015; Monereo \& Domínguez, 2014). Así mismo, destacamos la importancia de las relaciones que establece el profesor con los demás profesores, con sus voces, con sus historias y con la historia de la sociedad, ya que estas relaciones, consideramos, movilizan la actividad de enseñanza de los profesores (Morosini, 2000).

En esta misma línea de discusión, acerca de la formación de profesores que enseñan en la universidad a partir de la reflexión, Grillo (2000) comenta:

Las condiciones de enseñanza cambian día a día y no existe la seguridad de lo que "es correcto". En esta perspectiva, el profesor necesita ser un investigador que cuestione su pensamiento y su práctica, actúe reflexivamente en el ambiente dinámico, tome decisiones y cree respuestas más adecuadas porque son construidas en una situación concreta propia. (p. 75)

Para lograr esto, el profesor puede reflexionar continuamente sobre las condiciones en las cuales está realizando su actividad de enseñanza. Esto implicaría reconocer, como lo indica la autora, que el aula de clase es dinámica y que día a día supone nuevos retos.

A propósito de la necesidad de reflexionar sobre la propia práctica, retomamos a autores como Walker (2015), Vasconcellos y Sordi (2016), Silva y Davis (2016), Salas-Madriz (2016) 
y Moscoso y Hernández (2016), quienes discuten cómo los profesores que enseñan en la universidad hacen reflexiones pedagógicas, didácticas y metodológicas frente a asuntos propios de su actividad de enseñanza tales como preparación de clases, diseño curricular, evaluación y pautas a seguir para sus investigaciones.

Así mismo, Almeida y Pimienta (2014) reconocen el papel central de la reflexión en la formación pedagógica de los profesores que enseñan en la universidad. Estas autoras, al exponer una investigación realizada en una universidad, muestran la importancia que los profesores de algunos programas de pregrado le atribuían a la enseñanza basada en la reflexión. Vista así, la enseñanza universitaria puede adquirir más valor en tanto responda a reflexiones del profesor, las cuales parten de su reconocimiento como un sujeto inmerso en un contexto social, cultural, político y económico.

La misma necesidad de comprender los alcances de las actividades realizadas en el aula de clase por medio de la reflexión, y de tomar como punto de referencia el compartir de experiencias, es plasmada en la investigación de Campos, Dias y Silva (2016). Con estas autoras, reconocemos dos características importantes en el marco de la reflexión como posibilidad de formación continuada de profesores que enseñan en la universidad. En primer lugar, comprendemos que los saberes derivados de las experiencias de enseñanza pueden ser elementos de formación de otros profesores en espacios colaborativos. En segundo lugar, reconocemos que la reflexión sobre las acciones propias de sus actividades de enseñanza posibilita un ideario docente, en el cual el profesor se reconoce como profesional que no solo conoce una disciplina, sino que también estudia las posibilidades de enseñar a nivel didáctico y metodológico.

Otro elemento que resaltamos es la necesidad de que estas reflexiones sobre las propias actividades transformen, entre otras cosas, al conocimiento mismo. A propósito de esto, Grillo (2000) expone que

el profesor, como sujeto reflexivo que es, se inclina sobre el contenido de su propia experiencia, la examina, la relaciona con otras y la analiza a la luz de las experiencias propias de los otros. La experiencia actual aprovecha algo de las anteriores y contribuye para el perfeccionamiento de acciones posteriores, lo que defiende la idea de que el profesor debe construir su conocimiento por la reflexión sobre la práctica. (p. 76)

Este aporte muestra que la reflexión de los profesores ocupa un lugar importante en la toma de decisiones asociadas a la planificación de nuevas acciones en sus clases y en la creación de nuevo conocimiento disciplinar y pedagógico. Así mismo, advierte Grillo (2000), no se puede caer en modismos o en rutinas impuestas por las instituciones.

Con base en lo expuesto hasta aquí, expresamos algunas consideraciones respecto a la reflexión y el encuentro de voces de los profesores - en los colectivos de los que hacen parte- que enseñan en la universidad. En primer lugar, la formación continuada del profesor - que lo lleva a reflexionar sobre sus propias acciones- y el saber que construye en el aula de clase posibilitan una construcción de saberes que pueden generar cambios significativos, particularmente, enfocados en el encuentro entre la teoría y la práctica.

En segundo lugar, la enseñanza en la universidad va más allá de la transmisión de saberes e implica, entre otras cosas, una formación pedagógica continua, movilizada principalmente 
por la necesidad de organizar una enseñanza en la que coincidan el objeto de la actividad -la cual entendemos como la organización de la enseñanza- y el motivo que moviliza a este profesor.

En tercer lugar, a propósito de esta necesidad de organizar la enseñanza, consideramos que el profesor no puede recurrir únicamente a los saberes disciplinares para poder acompañar una clase, sino que también debe cuestionarse constantemente sobre la pertinencia de las metodologías que llevará al aula. Un camino para pensar estas metodologías es la reflexión colaborativa sobre la propia actividad de enseñanza.

\section{Metodología}

La investigación, como actividad que moviliza al sujeto y al objeto de conocimiento, demanda un método que oriente y dé sentido a las acciones en su interior. Así, al tener como fundamentación la teoría histórico-cultural, tomamos como referente metodológico el método dialéctico (Asbahr, 2011; Kopnin, 1966; Moretti, Martins \& Souza, 2016; Vygotski, 2009), el cual se caracteriza por los siguientes aspectos.

En primer lugar, en el método dialéctico reconocemos la historia y las condiciones concretas de actividad de los sujetos como constituyentes de su conciencia. La dialéctica como método define acciones que llevan al investigador a analizar procesos y no objetos estáticos. Así, el objeto de esta investigación es el proceso de organización de la enseñanza de tres profesores, Andrés, Jacinto y José del área de ciencias básicas de una institución universitaria.

En segundo lugar, el método dialéctico se caracteriza por usar técnicas e instrumentos de producción de registros y datos que no solo expliquen la naturaleza del objeto, sino que, además, lo transformen. De este modo, propusimos instrumentos y técnicas como entrevistas semiestructuradas, mapas de caso creados por los profesores y textos de estudios de caso escritos por los protagonistas de la investigación.

En tercer lugar, comprendemos las acciones y las voces de los protagonistas como la unidad de análisis de la investigación, de modo que, una vez revisados los datos producidos, mostramos a través de tres categorías el proceso de organización de la enseñanza de los profesores a través de estas voces y acciones. En este artículo, mostraremos una síntesis de una de estas categorías.

El trabajo de campo de la investigación tuvo tres momentos. En el primero, los profesores crearon un ideograma y un mapa conceptual en los cuales plasmaron su ideario (Jaramillo, 2003) sobre su propia actividad de enseñanza, la universidad y el conocimiento matemático. Esta actividad nos posibilitó comprender que la historia de vida de cada profesor, sus experiencias y procesos de formación inicial y continuada constituían su subjetividad como profesores de matemáticas que realizaban su trabajo en una institución universitaria de administración.

En el segundo momento del trabajo de campo, los profesores realizaron el proceso de escritura de estudios de caso, lo cual implicaba que comprendieran en qué consistía esta metodología para diseñar una estructura propia que orientara su actividad de creación de estudios de caso. Al terminar la escritura, los profesores socializaron algunos estudios 
de caso con sus colegas del área de ciencias básicas y, a partir del intercambio de voces y experiencias, refinaron los textos creados por ellos.

En el tercer momento, los profesores pusieron en escena los estudios de caso, de modo que sus estudiantes, a través del análisis de los textos creados, encontraron soluciones que mostraron cómo el conocimiento matemático posibilitaba comprender las realidades empresariales y mostraba caminos para tomar decisiones que beneficiaran a las organizaciones. Una vez terminadas las sesiones, cada profesor participó de una entrevista en la cual compartió sus impresiones sobre la experiencia vivida y los proyectos futuros en el marco de la creación de nuevos estudios de caso, pues, como los estudiantes y ellos mismos concluyeron, esta estrategia metodológica movilizó la percepción que tenían en cuanto al conocimiento matemático y a la necesidad de apropiarse de él para comprender el día a día de las empresas.

\section{El proceso de creación colectiva de estudios de caso}

La primera actividad en el proceso de creación de estudios de caso fue la participación de los profesores en una sesión de clase, en la que un profesor experto en estudios de caso les socializó los principales elementos de esta metodología. En esta clase, los tres profesores participaron como estudiantes y se hizo una lectura previa de un estudio de caso propuesto por el experto.

Durante la clase, el profesor experto utilizó la problemática de la empresa para crear preguntas problematizadoras que generaron el debate entre los participantes, el cual consistía en contrastar diferentes escenarios de solución para la empresa y validar, a través de cálculos, las consecuencias de las soluciones propuestas. Así mismo, el profesor experto compartió algunas estrategias para orientar una sesión mediada por estudios de caso, tales como el uso de varios tableros para sintetizar los datos del texto, el uso de esquemas para resumir la información más relevante, las preguntas antes, durante y después del debate, y el cierre por medio de una conclusión general sobre la solución más pertinente a la problemática analizada.

Al terminar la sesión, los profesores propusieron realizar lecturas de más estudios de caso, de modo que pudieran crear de manera colectiva un esquema en el cual plasmar sus ideas respecto a qué debía contener un estudio de caso en términos de estructura. Así, los profesores leyeron los casos Eastman Kodak Company: Funtime Film (Dolan, 2002), Cervecería 3 Cordilleras. Una idea que se convirtió en sueño; un sueño hecho realidad (Rave \& Franco, 2011), entre otros.

Al socializar sus hallazgos en cuanto a estructura, los profesores concluyeron que un estudio de caso comenzaba con unos párrafos introductorios, en los cuales el autor mostraba a nivel general cuál era la empresa, la problemática que enfrentaba y las consecuencias de la problemática para el presente y futuro de la empresa. La segunda parte del texto consistía en la descripción del contexto de la empresa, la cual, por lo general, contenía la historia de la organización, el portafolio de productos o servicios y una evaluación de su posición respecto a la competencia. Este relato del contexto, por lo general, presentaba la problemática de manera más específica. 
La tercera parte del texto consistía en una reseña de las variables que los estudiantes podrían tener en cuenta para analizar la problemática de la empresa y proponer soluciones. Por lo general, estas variables estaban asociadas a indicadores económicos, datos sobre competidores, análisis de oferta y demanda de los productos y perfiles psicológicos de los protagonistas del caso. La mayoría de los textos reseñados por los profesores terminaba con algunas preguntas problematizadoras o con el planteamiento del problema en términos de incertidumbre por parte de los personajes del texto.

A través de una lluvia de ideas colectiva, los profesores concluyeron que los estudios de caso que escribirían debían estructurarse de modo que presentaran los siguientes elementos. 1) la declaración de cuáles serían los temas y objetivos que se abordarían en el caso; 2) una introducción, en la cual se comentaría a grandes rasgos la problemática; 3) el contexto de la empresa, el cual mostraría a los estudiantes la posición de la empresa en el mercado; 4) las variables a analizar para comprender el problema y proponer soluciones; y 5) el planteamiento de la problemática del caso, a través de preguntas o la descripción de situaciones de incertidumbre. En la figura 1 mostramos el esquema construido por los profesores.

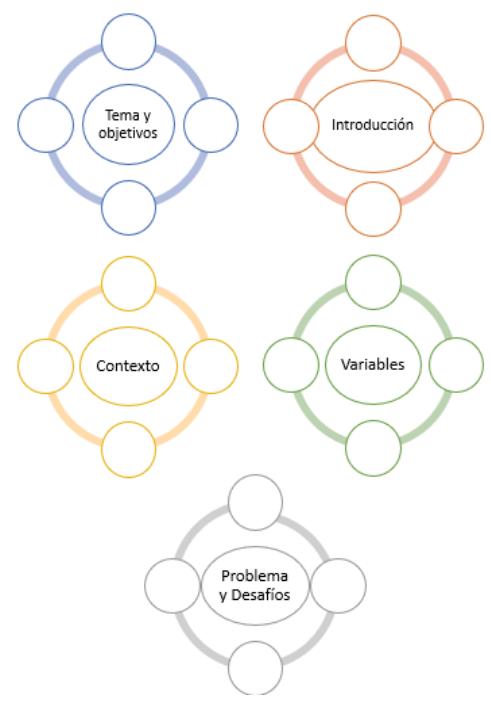

Figura 1. Estructura del mapa de caso construido por los profesores. Elaborado en la reunión de creación de esquema grupal (26 de octubre de 2018).

Una vez construido el esquema, los profesores crearon el primer mapa de caso con una temática propuesta por uno de ellos: el establecimiento del precio de venta de un producto. A continuación, mostramos cómo se dio la construcción de dicho mapa.

En primer lugar, los temas y objetivos del caso fueron el establecimiento del precio como función de la oferta y la demanda. Esta temática implicaba el conocimiento de funciones, variables económicas y la relación entre el precio de un producto y los costos fijos y variables de este.

En segundo lugar, los profesores acordaron que la empresa se llamaría Kelly's cupcakes, la cual se encargaba de vender cupcakes bajo demanda por medio de redes sociales. La problemática que sugirieron los profesores consistió en que los dueños de dicha empresa no se lograban poner de acuerdo en cómo establecer los precios de venta que les garantizaran una ganancia. 
En tercer lugar, los profesores hicieron una lluvia de ideas en cuanto al contexto de la empresa. En este sentido, la empresa Kelly's cupcakes quedó ubicada en la ciudad de Medellín - Antioquia-. Además, fue creada por una estudiante egresada de la institución universitaria donde trabajaban los profesores. La empresa se caracterizaba por tener precios competitivos en el mercado de ventas bajo demanda.

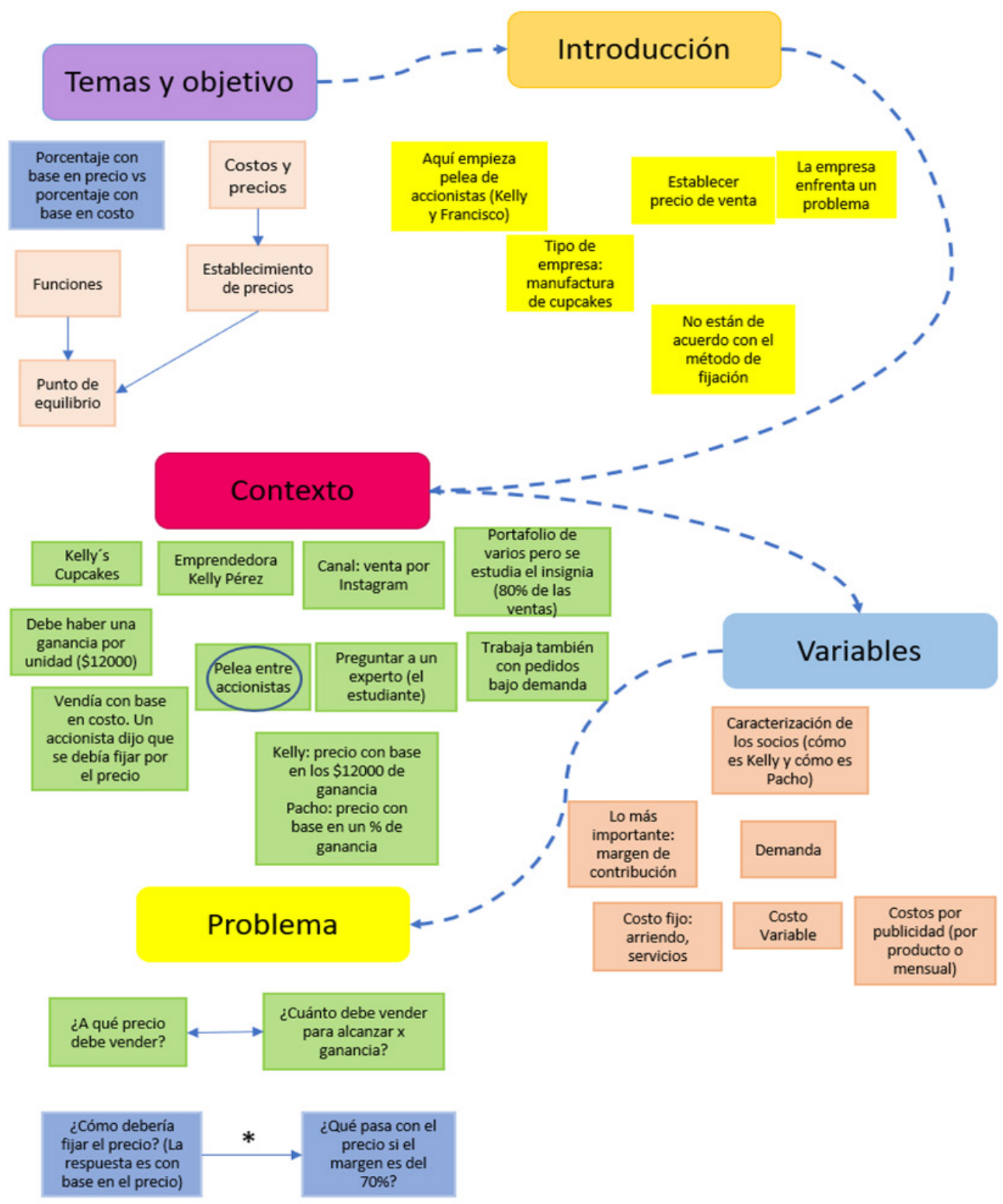

Figura 2. Mapa de caso construido colectivamente para la empresa Kelly's Cupcakes. Elaborado en la reunión de creación de estudio de caso grupal (19 de noviembre de 2019)

En cuarto lugar, las ideas propuestas en términos de variables del problema fueron: costos fijos, costos variables, ganancia esperada y características de los dueños de la empresa. Esta última variable fue importante para los profesores, pues, desde su punto de vista, la descripción de la personalidad de los dueños podría dar información implícita sobre el tipo de decisiones que ellos tomarían en cuanto al establecimiento del precio de venta.

Finalmente, los profesores propusieron problemas como: ¿a qué precio deben vender 
los cupcakes? ¿Cuántos cupcakes deben vender a ese precio para obtener las ganancias esperadas? ¿Cuál es el mejor método para establecer el precio de venta? Con estas preguntas, los profesores esperaban que los estudiantes analizaran, a la luz de los cálculos realizados, cuál era la mejor manera de establecer el precio de los cupcakes. En la figura 2 mostramos el mapa de caso creado de manera colaborativa por los profesores.

Respecto al camino recorrido por los profesores hasta aquí, resaltamos su postura activa para apropiarse de la metodología estudios de caso, pues, desde el primer encuentro, expresaron a través de sus voces la intención de construir una metodología que respondiera a sus propias expectativas y que estuviera también en consonancia con lo que la institución universitaria estableció en su modelo pedagógico.

En este sentido, el profesor, como sujeto activo que transforma la naturaleza a través de su trabajo, objetiva en la metodología al interior de la actividad de organización de la enseñanza la satisfacción de sus necesidades, lo cual demanda de entrada una postura activa y crítica de este sujeto, tal y como Moretti (2007) lo explica: "pensar la formación continuada de maestros pasa por viabilizar condiciones para que estos, movidos por las mismas necesidades de organizar la enseñanza, interactúen mediados por el objeto de su trabajo" (p. 28).

Además, observamos en las acciones de los profesores un interés por diseñar estudios de caso con una estructura propia, resultado de sus lecturas y comprensiones en términos de forma y contenido. A propósito, reconocemos que las condiciones concretas para la realización de la actividad de enseñanza posibilitaron que los profesores definieran una dialéctica entre la metodología de enseñanza - la forma- y el conocimiento matemático -el contenido- que fue socializado, construido y apropiado en el aula de clase. Esta necesidad de establecer la dialéctica entre estas dos categorías es explicada por Rosental y Straks (1960):

Toda forma concreta existe solamente en la medida en que corresponde total o parcialmente al contenido, en la medida en que abre ancho cauce a su desarrollo y contribuye al desarrollo del contenido, o, al menos, brinda una posibilidad de desarrollo por limitada que sea. (p. 224)

Así, consideramos que los profesores encontraron en los estudios de caso una posibilidad de llevar este conocimiento matemático de manera que los estudiantes, a través de sus reflexiones individuales y colectivas, encontraran en dicho conocimiento una forma de comprender el contexto en que se desenvuelven. Coincidimos en este sentido con Moura (2001), para quien

la naturaleza del conocimiento que el maestro deberá enseñar va a indicar una forma de relacionarse con los alumnos, de cómo organizar el espacio de aprendizaje, de cómo elegir los instrumentos que podrán propiciar un mejor aprendizaje de los contenidos a ser enseñados. (p. 147)

Al terminar el proceso de creación, conversamos con los profesores sobre las impresiones que les había dejado la discusión con la que se construyó el mapa:

Investigador: Ya después de haber hecho el esquema, ¿qué impresiones quedan? 
Andrés: Es muy práctico, porque es algo que hace uno todos los días, pero rayando en miles de hojitas, así como José ahí [en el mapa]. Digamos que es casi lo mismo, pero sintetizado de una manera muy corta y eficiente.

Investigador: ¿Esta forma de hacer las cosas está relacionada con la motivación para dar las clases? ¿Tiene que ver?

Andrés: Sí

Investigador: ¿Por qué?

Jacinto: Porque es algo que uno hace mental o indirectamente y puede ir dejando registro de eso. Hay cosas puntuales, por ejemplo, algunas de las variables que yo no habría tenido en cuenta, pero ya después de que las mencionó José, pues sí es válido.

Investigador: ¿Trabajo colaborativo?

Jacinto: Sí, porque todos manejamos el tema, todos sabemos de qué estamos hablando, pero cada uno puede pensar un pedacito distinto adicional. Entonces sería bueno recoger en un solo documento lo que vamos pensando los cuatro. Cuatro cabezas piensan más que una. (Creación mapa de caso grupal, 19 de noviembre de 2018)

De esta conversación retomamos dos aspectos fundamentales. En primer lugar, Jacinto resaltó la importancia de dejar registro de las ideas que se construyen de forma colectiva. En este sentido, la creación colectiva de los estudios de caso respondió a la necesidad de consolidar una metodología que se pusiera en escena en un momento específico, y pudiera, además, servir como referente para posteriores encuentros con los estudiantes. Así, Jacinto, al afirmar: "todos manejamos el tema, todos sabemos de qué estamos hablando, pero cada uno puede pensar un pedacito distinto" y añadir que "cuatro cabezas piensan más que una", resaltó la importancia de tener en cuenta las voces y experiencias de los demás integrantes del colectivo. A propósito, Fiorentini, Souza y Melo (2000) comentan:

Aunque el saber sea personal y evolucione con el tiempo y la experiencia, él es cultural, es decir, se constituye por la interacción con los otros miembros de nuestra cultura. Nuestro saber no es aislado, él es compartido y se transforma, se modifica a partir del intercambio de experiencias y de la reflexión colectiva con los otros. (p. 322)

En segundo lugar, el proceso de creación colectiva que se sintetizó en un solo documento - como mencionó Jacinto- demostró que las diversas ideas respecto al mismo contenido se podían estructurar en la misma forma. Así mismo, consideramos importante resaltar que esta consolidación se dio en el marco de condiciones concretas particulares. Desde nuestro punto de vista, la dialéctica entre contenido y forma se fue estableciendo cuando, en la actividad de organización de la enseñanza, se reconocieron los saberes que, a través de la discusión con los estudios de caso, iban a aportar los estudiantes a la construcción y apropiación colectiva en el aula de clase, en un contexto real, al reconocer problemáticas reales de las organizaciones.

Con estas impresiones en mente, durante la siguiente semana nos reunimos con los profesores para escribir el borrador del texto de estudio de caso, teniendo como referencia el mapa de caso que habíamos creado. En este texto tuvimos en cuenta aspectos como los mencionados por Wassermann (1994), quien argumenta que un texto de estudio de caso debe motivar la lectura a través de la descripción de posturas activas de los protagonistas. 
Así,

a diferencia de otros tipos de relato, que pueden tener un comienzo lento o incluir pasajes descriptivos, los casos, por lo general, empiezan refiriendo una acción. La idea es atrapar la atención del lector a fin de que los alumnos no reaccionen diciendo para sí "Hmm. Otra tarea aburrida”, sino diciendo “CCaramba! ¿Qué es esto? ¡Quiero saber más!”. (p. 71)

Durante los encuentros en los que creamos el texto de este estudio de caso, los profesores sugirieron que adjuntáramos anexos con datos de las ventas por internet en Colombia, de modo que los estudiantes identificaran aspectos del contexto de la empresa de Kelly y Francisco que sirvieran para que sus análisis estuviesen más fundamentados. Los profesores sugirieron dos aspectos puntuales: el texto debía estar escrito en pasado y debía hacer explícita la necesidad de que la empresa no se viese en una situación de inestabilidad financiera, dado que era el proyecto de empresa de una persona recién egresada de un programa de administración de empresas, lo cual motivaría a los estudiantes a utilizar estos razonamientos matemáticos en sus propios contextos.

Luego de tener el primer borrador del estudio de caso escrito de manera colectiva, los profesores y los investigadores nos reunimos para revisar la estructura y la redacción. Al terminar la lectura individual, les preguntamos a los profesores sus impresiones sobre el texto de estudio de caso creado:

Investigador: ¿Cómo ven la estructura?

Jacinto: Yo me sentí leyendo el caso Kodak.

Investigador: ¿Por qué?

Jacinto: Me sentí en Harvard, leyendo un artículo de Harvard.

Andrés: Yo lo vi muy bien.

José: Yo lo veo bien.

Investigador: ¿Lo ven viable para escribirlo? ¿Se ven escribiendo un caso así?

Andrés: Yo sí, pero ayudado.

Investigador: Dentro del proceso está el hacer el mapa colectivamente y después hacer un borrador con cada uno y después socializarlo y llevarlo a la clase. La idea es llevar uno a clase en el primer y segundo periodo del año entrante. (Socialización estudio de caso grupal, 26 de noviembre de 2018)

Las respuestas de los profesores a la pregunta sobre sus percepciones en cuanto al proceso de escritura dan cuenta de la importancia de la colaboración en los procesos de creación de metodologías para llevar al aula de clase el conocimiento creado culturalmente. Así, cuando Andrés dice "yo sí [escribiría el caso], pero ayudado", da cuenta de una necesidad de escuchar los aportes de los demás integrantes del colectivo para que el objeto de su actividad de enseñanza se fundamente en procesos de reflexión colectiva sobre su propia actividad. En este sentido, coincidimos con Imbernón (1994), para quien

la práctica educativa se concibe básicamente como un proceso de reflexión, de análisis, de indagación y de mejora a través de la autoevaluación, lo que nos viene a decir que, en 
el centro, desde esa práctica, aparece la reflexión y la autoevaluación aplicada a la propia acción docente. (p. 94)

Cuando Jacinto dice "me sentí en Harvard, leyendo un artículo de Harvard", él da cuenta de que el producto de esta actividad de organización de la enseñanza cumple con los criterios que la institución universitaria ha establecido como pertinentes para la construcción de conceptos para la solución de problemas empresariales. Consideramos entonces que los profesores protagonistas encontraron en la actividad de creación colectiva de estudios de caso una posibilidad para el encuentro entre el sentido personal que le atribuyen a su actividad de enseñanza y el significado que la institución universitaria ha construido en torno a la actividad de enseñanza que han privilegiado a través de su modelo pedagógico.

\section{Conclusiones}

Nuestro objetivo en este artículo fue mostrar el proceso de organización de enseñanza de tres profesores que enseñan matemáticas en una institución universitaria de administración, a partir de la creación colectiva de estudios de caso. Para lograrlo, mostramos los fundamentos teóricos y epistemológicos que orientaron la investigación doctoral que estamos reseñando, así como las fases, objeto y unidad de análisis en el marco del método dialéctico.

Al narrar el proceso de creación del primer estudio de caso por parte de los profesores, el camino recorrido nos posibilita cerrar este artículo con algunas reflexiones.

En primer lugar, el método dialéctico como orientador de las actividades a desarrollar con los profesores en la investigación posibilitó comprender el movimiento de sus acciones. En este sentido, la dialéctica como lógica que fundamenta al método nos brindó elementos para analizar la movilización de la actividad de enseñanza de los profesores, mientras se recuperaban aspectos asociados con su historicidad.

En segundo lugar, observamos en las acciones y las voces de los profesores una postura sobre el conocimiento matemático que lo caracteriza como una herramienta para comprender las condiciones de vida de los sujetos en sus actividades sociales y culturales. Así, las matemáticas superaron el carácter teórico y estático y se constituyeron como una posibilidad para analizar el entorno, comprender las problemáticas - en este caso de una empresa- y ofrecer escenarios de solución.

En tercer lugar, comprendemos que el proceso de escritura de los profesores protagonistas estuvo permeado por una intencionalidad de crear, a partir de sus propias necesidades, experiencias y saberes, una forma propia de mostrar el conocimiento matemático. En este sentido, queremos llamar la atención sobre el papel que el mapa de caso ejerció durante todo el proceso de escritura del caso, pues los profesores destacaron cómo este mapa, creación suya, aportó con su estructura a un entendimiento de qué era lo que debían escribir.

Finalmente, encontramos en el proceso de creación de los profesores un movimiento constante de sus idearios sobre el conocimiento matemático, la universidad y su modelo pedagógico y sus estudiantes, a través de sus voces y experiencias. Esta forma de verse a ellos mismos, a las matemáticas y a su contexto respondió a un proceso de reflexión sobre sus actividades, aspecto que, como mencionamos anteriormente, moviliza a los profesores 
en la búsqueda de nuevas apuestas metodológicas para la apropiación del conocimiento que ha sido construido histórica y socialmente.

\section{Referencias}

Almeida, M. (2012). Desenvolvimento profissional dos docentes do ensino superior. Contributos para a compreensão do desenvolvimento profissional dos docentes que atuam na formação inicial de professores (Tesis doctoral inédita, Universidade de Lisboa, Lisboa, Portugal).

Almeida, M., \& Pimenta, S. (2014). Pedagogia universitária - Valorizando o ensino e a docência na universidade. Revista Portuguesa de Educação, 27(2), 7-31.

Araujo, E. (2003). Da formação e do formar-se. A atividade de aprendizagem docente em uma escola pública (Tesis doctoral inédita, Universidade de São Paulo, São Paulo, Brasil).

Arias, F., Betancourt, J., Garcés, L., Echeverri, C., \& Quirama, U. (2017). Casos de enseñanza como estrategia pedagógica en programas empresariales. Civilizar Ciencias Sociales y Humanas, 17(33), 243-256. https://doi.org/10.22518/usergioa/jour/ccsh/2017.2/a14

Asbahr, F. (2011). Por que aprender isso, professora? Sentido pessoal e atividade de estudo na Psicologia histórico-cultural (Tesis doctoral no publicada, Universidade de São Paulo, São Paulo, Brasil).

Caballero, K., \& Bolívar, A. (2015). El profesorado universitario como docente: hacia una identidad profesional que integre docencia e investigación. REDU. Revista universitaria de educación, 13(1), 57-77.

Cadavid, L. (2017). Constitución de la subjetividad del sujeto maestro que enseña matemáticas, desde y para la actividad pedagógica (Tesis doctoral inédita, Universidad de Antioquia, Medellín, Colombia).

Campos, V., Dias, M., \& Silva, A. (2016). Constitução da identidade profissional docente e as ações formativas na universidade federal de Uberlândia-MG. XIII Seminário Nacional O Uno e o Diverso na Educação Escolar e XVI Semana da Pedagogia (pp. 2588-2604). Uberlândia: Universidade Federal de Uberlândia.

Cedro, W. (2016). Changing teachers' mathematical knowledge during their teaching activity. RIPEM, 6(2), 89-110.

Dolan, R. (2002). Eastman Kodak Company: Funtime Film (Caso HBS 9-594-111). Boston: Harvard Business School.

Ellet, W. (2007). The case study handbook. How to read, discuss, and write persuasively about cases. Boston, Estados Unidos: Harvard Business Review Press.

Ellet, W. (2008). The case study handbook. A student's guide. Boston, Estados Unidos: Harvard Business Review Press.

Fiorentini, D., \& Miorim, A. (2001). Pesquisar \& escrever também é preciso: a trajetória de um grupo de professores de matemática. En D. Fiorentini, \& A. Miorim (Eds.), Por trás da porta, que matemática acontece? (pp. 17-44). São Paulo, Brasil: Cempem. 
Fiorentini, D., Souza, A., \& Melo, G. (2000). Saberes docentes: um desafio para acadêmicos e práticos. En C. Geraldi, D. Fiorentini, \& E. Pereira (Eds.). Cartografías do trabalho docente (pp. 307-335). São Paulo, Brasil: Mercado de letras.

Grillo, M. (2000). O lugar da reflexão na construção do conhecimento profissional. En M. Morosini, Professor de ensino superior. Identidade, docência e formação (pp. 75-80). Brasília, Brasil: Instituto Nacional de Estudos e Pesquisas Educacionais.

Imbernón, F. (1994). La formación y el desarrollo profesional del profesorado. Hacia una nueva cultura profesional. Barcelona: Graó.

Jaramillo, D. (2003). (Re)constituição do ideário de futuros professores de Matemática num contexto de investigação sobre a prática pedagógica (Tesis doctoral inédita, Universidade Estadual de Campinas, Campinas, Brasil).

Jiménez, A. (2005). Formación de profesores de matemática: aprendizajes recíprocos escuelauniversidad. Tunja, Colombia: Universidad Pedagógica y Tecnológica de Colombia.

Kopnin, P. (1966). Lógica dialéctica. México, D. F.: Editoral Grijalbo S. A.

Leontiev, A. (1988). Uma contribuição à Teoria do desenvolvimento da Psique infantil. En L. Vygotski, A. Luria, \& A. Leontiev. (Eds.), Linguagem, desenvolvimento e aprendizagem (pp. 59-83). São Paulo, Brasil: Ícone.

Leontiev, A. (2004). O desenvolvimento do psiquismo. São Paulo, Brasil: Centauro Editora.

Mazo, D. (2011). Lideramos la educación virtual en Colombia. Lecciones aprendidas. Bogotá, Colombia: Comunicaciones \& Publicidad.

Migueis, M. (2010). A formação como actividade de aprendizagem docente (Tesis doctoral inédita, Universidade de Aveiro, Aveiro, Portugal).

Monereo, C., \& Domínguez, C. (2014). La identidad docente de los profesores universitarios competentes. Educación XXI, 17(2), 83-104. https://doi.org/10.5944/educxx1.17.2.11480

Moretti, V. \& Moura, M. (2010). O sentido em movimento na formação de professores de matemática. Zetetiké, 18(34), 155-180. https://doi.org/10.20396/zet.v18i34.8646682

Moretti, V. (2007). Professores de matemática em atividade de ensino. Uma perspectiva histórico-cultural para a formação docente (Tesis doctoral inédita, Universidade de São Paulo, São Paulo, Brasil).

Moretti, V., Martins, E., \& Souza, F. (2016). Dialectical and historical method, culturalhistorical theory and education: some appropriation in research on education of teachers who teach mathematics. RIPEM, 6(2), 54-72.

Morosini, M. (2000). Docência universitária e os desafios da realidade nacional. En M. Morosini, Professor do ensino superior: identidade, docência e formação (pp. 11-20). Brasilia, Brasil: Instituto Nacional de Estudos e Pesquisas Educacionais.

Moscoso, F., \& Hernández, A. (2015). La formación pedagógica del docente universitario: un reto del mundo contemporáneo. Revista Cubana de Educación Superior, (3), 140-154.

Moura, M. (2001). A atividade de ensino como ação formadora. En A. Castro, \& A. Carvalho 
(Orgs.), Ensinar a ensinar: didática para a escola (pp. 142-161). São Paulo: Editora Pioneira.

Polettini, A. (2000). Mathematics teaching life histories in the study of teachers' perceptions of change. Teaching and Teacher Education, 16, 765-783. https://doi.org/10.1016/S0742051X(00)00024-X

Puchol, L. (2005). Nuevos casos en dirección y gestión de recursos humanos 25 casos de recursos humanos acompañados de las soluciones propuestas por sus autores. Madrid: Díaz de Santos.

Radford, L. (2016a). On alienation in the mathematics classroom. International Journal of Educational Research, 79, 258-266. https://doi.org/10.1016/j.ijer.2016.04.001

Radford, L. (2016b). The theory of objectification and its place among sociocultural research in mathematics education. RIPEM, 6(2), 187-206.

Rave, E., \& Franco, J. (2011). Casos empresariales colombianos. Decisiones gerenciales ante momentos de crisis. Sabaneta, Colombia: Ceipa Business School.

Rigon, A., Asbahr, F., \& Moretti, V. (2010). Sobre o processo de humanização. En M. Moura (Ed.), A atividade pedagógica na teoria histórico-cultural (pp. 13-44). São Paulo, Brasil: Liber Livro.

Rosental, M., \& Straks, G. (1960). Categorías del materialismo dialéctico. México D.F.: Grijalbo.

Salas-Madriz, F. (2016). Aportes del modelo de Yrjö Engeström al desarrollo teórico de la docencia universitaria. Revista Educación, 40(2), 1-22.

Silva, V., \& Davis, C. (2016). Contribuições metodológicas para a análise dos sentidos em um estudo sobre atividade docente. Educação e Pesquisa, 42(1), 39-51. https://doi. org/10.1590/S1517-9702201603125797

Stößlein, A., \& Kanet, J. (2016). Undergraduate research experiences: Identifying lessons learned and challenges for business schools. The International Journal of Management Education, 14, 349-367. https://doi.org/10.1016/j.jime.2016.07.004

Vasconcellos, M., \& Sordi, M. (2016). Formar professores universitários: tarefa (im) possível?. Interface. Comunicacão, saúde, educacão, 20(57), 403-414. http://dx.doi. org/10.1590/1807-57622015.0450

Vygotski, L. (2001). A construção do pensamento e da linguagem. São Paulo, Brasil: Martins Fontes.

Vygotski, L. (2009). El desarrollo de los procesos psicológicos superiores (2ª ed.). Barcelona, España: Crítica.

Walker, V. (2015). Aportes teóricos para pensar el trabajo docente en la universidad. Revista electrónica Actualidades investigativas en educación, 15(1), 1-20. http://dx.doi. org/10.15517/aie.v15i1.16964

Wassermann, S. (1994). El estudio de casos como método de enseñanza. Buenos Aires, Argentina: Amorrortu Editores. 